\title{
Resumen de Actividades del Comité de Tuberculosis. Región Metropolitana, 2005
}

\author{
Activities of Tuberculosis Committee of Santiago during 2005. A summary
}

\section{Marzo}

\section{Actividades 2004 y discusión de temas pendientes}

Drs. Carlos Peña M. y Manuel Zúñiga G.

- La Ley de Autoridad Sanitaria y su reglamento ya está en discusión en la Contraloría.

- Análisis de las Nuevas Normas Técnicas del Progama de Control de la Tuberculosis (PCT).

- Limitantes actuales en la gratuidad y hospitalización de los pacientes tuberculosos.

- Nueva estructura de salud: La Subsecretaria de Salud Pública generará un Departamento de Prevención y Control de Enfermedades con un Sub Departamento de Enfermedades Transmisibles donde se encuentra el PCT. A Nivel Central existirá un Director del "Programa de Control y Erradicación de la Tuberculosis" (PROCET), con una enfermera coordinadora nacional. Además, se crean tres tipos de comités de apoyo al PCT:

a) Comités de Gestión (Comités Regionales);

b) Comités Técnicos-Científicos (en el Instituto Nacional del Tórax), y

c) Comité de Laboratorios (en el Instituto de Salud Pública).

A Nivel Intermedio se mantiene la actividad gerencial de recursos, capacitación y otros a través de los "Equipos Técnicos de Tuberculosis" (ETT).

La SEREMI controlará los recursos de fármacos y de laboratorio así como la gestión de recursos de los servicios.

Se refuerza normativa de vigilancia epidemiológica (Ord. 135): Las notificaciones deben ser enviadas a la SEREMI a través del Boletín de Enfermedades de Notificación Obligatorias (ENO) y no pasaran por el PCT, sino que por los Servicios.

Se analiza el déficit de enfermeras del PCT de los Servicios de Salud Norte, Oriente, Central y Sur.

Se presenta un artículo que comunica una incidencia mayor de tuberculosis en los adultos fumadores.

\section{Abril}

Funciones del SEREMI y del PCT

Drs. Carlos Peña M. y Manuel Zúñiga G.

- Se informa sobre la distribución de funciones del SEREMI y del PCT.

- La subsecretaria de Salud Pública solicitó la ratificación de los integrantes de los equipos intermedios de los Servicios, debiendo reiterarse la descripción de los roles de sus integrantes para que los directivos locales reconozcan sus actividades.

- Las funciones del nivel intermedio son:

\section{1) Nivel de Secretarías Regionales}

Ministeriales (SEREMIS)

Los encargados de la tuberculosis en las SEREMIS serán responsables de:

a) Efectuar la evaluación epidemiológica y operacional.

- Consolidar trimestralmente información operacional mensual sobre morbilidad tuberculosa, recolectada de los Servicios de Salud.

- Anualmente efectuarán la evaluación epidemiológica y diagnóstica de situación y redactarán el texto anual de Evaluación del PCT, que será enviado a la Unidad de Tuberculosis del MINSAL, a través del Secretario Regional.

b) Ejercer las Actividades de Autoridad Sanitaria. 
- Velar por el adecuado cumplimiento de las actividades a nivel de la red asistencial.

- Establecer el contacto y coordinación a nivel regional con instituciones, organizaciones, centros y unidades de atención privada con el fin de precisar grado de conocimiento, aceptación y calidad en el cumplimiento de las normas técnicas y administrativas del PCT.

- Mantener al día la información sobre la Red de laboratorios públicos y laboratorios privados que participan de las actividades del PCT.

c) Asegurar la logística del PCT, para mantener su continuidad.

- Deberán conocer mensualmente el nivel de saldos de medicamentos con el objeto de suplir toda deficiencia ocasional, a través de transferencias interregionales o adquisición.

d) Como coordinador regional debe promover la realización de reuniones mensuales o bimestrales de los integrantes de los ETT, que operan a nivel de la Dirección de Servicios en cada región, originando los Comités Regionales de Tuberculosis.

\section{2) Nivel de Dirección de Servicios}

a) Ubicación y constitución.

- El director del Servicio de Salud (DSS), delegará en el Jefe del Departamento Técnico de Salud o su equivalente la responsabilidad del control de la tuberculosis. Para estos efectos propondrá el nombramiento por resolución del DSS de un "Equipo Técnico de Tuberculosis"(ETT) que cumplirá funciones de asesoría, coordinación y de referencia técnico-administrativo.

- El ETT estará integrado por un médico con adecuada formación y experiencia clínica, epidemiológica y operacional en el del Control de la Tuberculosis; una enfermera con conocimientos y práctica en las actividades técnico-administrativas del Control de la Tuberculosis, y un profesional de laboratorio (tecnólogo médico u otros) con experiencia en bacteriología de la tuberculosis y en los aspectos técnicos administrativos que requiere el funcionamiento de la red de laboratorios del PCT.

- Es recomendable que el horario asignado al médico y a la enfermera para sus actividades en el ETT, dependa de la población cubierta, y de la complejidad y extensión de la red asistencial. En términos generales, los servicios que cubran $>800.000$ habitantes reque- rirán dedicación a tiempo completo para esta actividad, con sede en la dirección del Servicio.

El encargado de las actividades de laboratorio será el responsable del laboratorio de referencia de tuberculosis del Servicio (laboratorio tipo II), es recomendable que dedique al menos $11 \mathrm{~h} /$ semana a esta actividad.

- Los ETT de los Servicios serán los interlocutores válidos y habituales del PCT a nivel de las SEREMIS y del PROCET nacional cuando sea necesario.

\section{3) Funciones específicas}

a) Mantener la estructura de gestión del PCT en el Servicio de Salud asegurando su adecuada situación estructural.

- Mantener al día el cuadro de profesionales capacitados en tuberculosis en cada servicio y asegurar el oportuno reemplazo o substitución de los profesionales que abandonan.

- Proponer al nivel central los nombres de los profesionales que requieren capacitación en Epidemiología y Control de Tuberculosis.

- Realizar el diagnóstico de situación de la tuberculosis y su control en la red asistencial del respectivo servicio de salud y proponer, los planes y estrategias de control, así como programar las actividades correspondientes.

- Informar al Nivel Central por medio del Nivel Regional sobre la marcha del programa.

- Constituirse en el referente técnico-administrativo del PCT frente a los equipos de salud que actúan a Nivel Local.

- Consolidar la información regional sobre las necesidades de medicamentos e insumos de laboratorio.

- Informar en forma oportuna y documentada a los responsables regionales de situaciones o hechos que corresponda conocer a ese nivel para que en su calidad de autoridad sanitaria, tome las acciones pertinentes.

\section{b) Capacitación}

- Conocer, calificar y autorizar las actividades de capacitación efectuadas a nivel de cada servicio. Promover y en caso necesario organizar cursos o actividades de capacitación con proyección regional.

\section{c) Investigación}

- Identificar áreas que requieran investigación epidemiológica u operacional sobre tuberculosis.

- Conocer y calificar todo proyecto de investi- 
gación cooperativa relacionada con tuberculosis.

- Proponer y efectuar investigaciones operacionales, con la aprobación del nivel regional.

\section{4) Actividades específicas del Equipo Técnico de Tuberculosis (ETT)}

\section{a) Funciones del médico:}

i) Es el responsable de las actividades de control; ii) Efectuar auditorías de diagnóstico, tratamiento y de mortalidad, enviando la respectiva información al nivel central, a través del nivel regional; iii) Realizar anualmente el estudio de una cohorte de pacientes "antes tratados" (AT), según Normas del PCT; iv) Constituir con neumólogo y neumopediatra equipos de referencia clínicos para resolver problemas diagnósticos y terapéuticos; v) Organizar y llevar a cabo, junto a los otros integrantes del ETT, las actividades de gestión del programa (supervisión, capacitación, evaluación, vigilancia epidemiológica, programación y actividades de promoción). Realizar la notificación y seguimiento de pacientes multiresistentes al MINSAL en los formularios normados.

\section{b) Funciones de la enfermera}

i) Participar en las actividades de gestión del PCT; ii) Efectuar la supervisión directa e indirecta y asesoría de las actividades de localización de casos, diagnóstico y tratamiento. Controlar el nivel de funcionamiento técnico-administrativo del tratamiento supervisado; iii) Verificar nivel de existencias, control de distribución y consumo de los medicamentos que utiliza el programa; iv) Efectuar auditorías de abandonos, enviando la respectiva información a nivel ministerial a través del nivel regional; v) Llevar el registro de pacientes extranjeros aplicando la encuesta de pacientes extranjeros; vi) Mantener una base de datos a través del Registro Nacional con el cruce de la información de notificaciones, tarjetero casos activos y registro de casos; vii) Depurar la información e informar mensualmente al nivel central a través del nivel regional; viii) Organización y seguimiento del estudio de contactos; ix) Realizar dos cohortes de casos nuevos (VT) y comunicarlos al nivel central por medio del nivel regional.

\section{c) Funciones del Profesional del Laboratorio}

i) Participar en las actividades del PCT; ii) Controlar el nivel de funcionamiento técnico y articular de la red de laboratorios que participan en las actividades de control; iii) Verificar la co- rrecta utilización de los sistemas de registro de actividades bacteriológicas; iv) Efectuar la supervisión técnica directa e indirecta (control de calidad); v) Organizar y llevar a cabo las actividades de capacitación y adiestramiento en bacteriología de la tuberculosis; vi) Mantener actualizada la información sobre nivel y calidad de instrumental, equipamiento e instalaciones de laboratorio; vii) Mantener le relación técnico administrativa con la Sección Micobacterias del Instituto de Salud Pública.

\section{5) Relaciones institucionales}

a) Dirección Regional de Gendarmería: Informe mensual de casos de tuberculosis ingresados y tratados, así como actividades de capacitación.

b) Instituto de Salud Pública: Informe de Red de laboratorios públicos y privados que realizan exámenes microbiológicos de detección de la tuberculosis.

c) Oficina de Profesiones Médicas: Coordinación con instituciones de salud privadas con fines de difusión de las actividades del programa.

\section{6) Principales dificultades en el proceso de reorganización}

a) Cambios en el flujograma de notificación (decreto Ley 158 de Octubre 2004): Los boletines ENO se enviarán desde el establecimiento donde se notifica en forma directa a la Unidad de Epidemiología de la SEREMI.

b) Equipos ETT incompletos: Lo que dificultan la coordinación para traslados y actualizaciones de información para Registro Nacional. Esto es más crítico en el Servicio Metropolitano Norte.

\section{Mayo}

Plan de Trabajo de la Unidad de Tuberculosis del Subdepartamento de Planificación de la SEREMI de Salud de la Región Metropolitana (RM).

Como Autoridad Sanitaria esta entidad debe velar por el cumplimiento del PCT, enfatizando su papel de supervisión.

\section{Plan de trabajo:}

a) Evaluación epidemiológica y operacional, identificando grupos de riesgo.

b) Consolidar y analizar la información de los seis Servicios de Salud de la RM, enviando el consolidado al Nivel Central. 
c) Mantener actualizado el Registro Nacional, enviando el consolidado al Nivel Central.

d) Disponer de un Registro Nacional de Auditorías de muertes, abandonos y encuesta de extranjeros.

e) Coordinar y analizar la cohorte de casos nuevos (VT) y antes tratados (AT) de los seis Servicios de Salud.

f) Actualizar la Base de Datos de Multiresistencia.

g) Velar por la utilización adecuada de los fondos para la adquisición de insumos y equipos de laboratorio con dineros de la campaña sanitaria.

h) Conocer y mantener actualizado el saldo mensual de medicamentos antituberculosos.

i) Consolidar la información de los 6 servicios en relación a medicamentos y envío de la información al nivel central.

j) Colaborar en actividades de capacitación.

k) Aplicar las pautas de supervisión a los Servicios.

l) Coordinación con el Sanatorio San José de Maipo para facilitar los traslados y disponer de un informe mensual de ingresos y egresos.

\section{Junio}

\section{Estructura y Función del Programa de Control de la Tuberculosis}

Dr. Carlos Peña M.

1) "Unidad de Control y eliminación de la tuberculosis" (PROCET), dentro de la Sección de Enfermedades Transmisibles del MINSAL, es equivalente al nivel central.

2) Unidad de la Tuberculosis en SEREMI. Está a cargo de la Coordinación Regional, vigilancia de actividades e indicadores y de la actividad intersectorial con instituciones privadas.

3) Comités Regionales con las asociación de ETT y SEREMI.

4) Se reitera la mantención de la gratuidad para el diagnóstico y tratamiento de la TBC, aunque existe déficit de precisión en las coberturas e indicadores de hospitalizaciones.

5) Información de las tasas actuales de tuberculosis:

En 2003, Chile tenía una incidencia de 18,3/ 100.000 hbs y en 2004 de 17,2/100.000 hbs. Además, el $75 \%$ de la población se encuentra con tasas menores de 20/100.000 hbs. Cuatro servicios con 3,5 millones habitantes, bajo 10 y 12 servicios con 8 millones de habitantes con tasas entre 15 y 16 . Sólo 3 servicios se encuentra en situación alarmante: Arica e Iquique con tasas sobre 30 y Osorno con tasas entre 27 y 28 , pero estos sólo representan el 4\% de la población nacional (600.000 habitantes).

Respecto a la monitorización del tratamiento, se concluye que el mejor predictor de abandono es el enfermo que comienza a fallar precozmente. En este caso se debe tener "tolerancia cero" y emplear los mayores recursos educativos y de motivación. Se señala como efectiva la aplicación del "puntaje de riesgo de abandono" que ha permitido reducir los abandonos de $8 \%$ a $4 \%$, por lo que se insistirá en que todos los Servicios lo apliquen.

\section{Julio}

\section{Encuestas destinadas a reconocer aspectos críticos del PCT}

Dr. Fernando Tabilo P., SSMSO

Se analiza una serie de encuestas efectuadas para reconocer aspectos críticos del PCT: a) demoras de los pacientes en consultar; b) demoras en hacer el diagnóstico; c) grado de conocimiento sobre la enfermedad y sobre el PCT por parte de los enfermos y del equipo de salud.

La demora en consultar de los enfermos bacilíferos con tos durante más de dos semanas es de alrededor de 2 meses. En ese lapso ya se ha producido contagio intrafamiliar. La demora en consultar depende de las creencias y percepciones del grado de amenaza que represente la enfermedad. Por ello se diseñó una encuesta para identificar las barreras personales del acceso al PCT.

Demora en el diagnóstico: Sólo el 24\% de los casos son diagnosticados en la primera consulta en los policlínicos y una proporción similar ocurre a nivel de neumólogos (21\%). Se destaca el aporte del diagnóstico de una segunda consulta $(10 \%)$ y de las actividades de enfermería (13\%).

En definitiva el diagnóstico médico ocurre en el $64 \%$ de ellos y se efectúa en consultorios en $40 \%$ de los casos. El 56\% de los pacientes diagnosticados estaba en control médico regular por otra patología (29\% por diabetes mellitus; $21 \%$ EPOC, y $17 \%$ programa VIH). Dos de cada tres médicos no lograron reconocer la tuberculosis en la primera consulta.

Es importante destacar que las enfermeras aportan uno de cada 3 casos bacilíferos y que existe una proporción importante de diagnósticos hechos por los Servicios de Urgencia (23\% SAPU y Postas) y $19 \%$ por médicos particulares. 
Se debe mejorar el conocimiento que existe de la enfermedad entre los médicos. Un 53\% de los consultados dice que la tuberculosis aumentó por efecto del SIDA; sólo un $28 \%$ dice saber que la tasa actual es menor de 20/100.000 hbts. La mayoría cree que no es posible erradicar la enfermedad y no conocen los beneficios del PCT ni sus actividades. Un $26 \%$ no pide baciloscopías a los sintomáticos respiratorios y la mayoría sobreestima el valor diagnóstico de la radiografía de tórax.

\section{Agosto}

\section{Análisis de la Tuberculosis en los hospitales Dra. Paulina Ramonda C., SSMSur}

Se relata el análisis de siete hospitales supervisados que aportan 200 casos nuevos anualmente, $40 \%$ de la Atención Primaria y $18 \%$ de especialidades (CDT).

Según los datos analizados se concluye que existe un déficit de notificación y falta de referentes de tuberculosis en los hospitales.

Se realizaron reuniones con los directores de los hospitales y el ETT, entregándose información sobre el PCT, la pesquisa y el estado actual de la enfermedad en el país, tendiente a sensibilizar y a mejorar la cooperación de estos niveles.

La tuberculosis en los hospitales es un tema interesante ya que son muchos los tópicos a revisar, destacando los siguientes:

a) Criterios de hospitalización: hemoptisis, reacciones adversas medicamentosas severas, descompensaciones agudas, comorbilidades, fracaso terapéutico, etc.

b) Oportunidad y calidad de las notificaciones (ENO) intrahospitalarias.

c) Medidas adecuadas de aislamiento.

d) Estudio de contactos intrahospitalarios.

e) Cumplimiento de normas terapéuticas y validez de las terapias empíricas.

f) Personal de enlace responsable de los traslados.

\section{Septiembre}

\section{Nuevas Normas del PCT}

\section{Drs. Fernando Tabilo P. y Carlos Peña M.}

Se destaca en estas nuevas normas (las anteriores datan de 1996) una actualización epidemiológica, una estructura acorde a los nuevos planes de Salud, con mayor precisión sobre los sistemas de registro y definición de roles y actividades de los distintos niveles.

Se incluye, además, un capitulo novedoso de Normas de Bioseguridad y se definen metas en relación a las estrategias frente a la enfermedad en etapas de eliminación avanzada: tasa de tuberculosis en todas las formas de 10/100.000 habitantes (hbts), susceptible de lograr a fines del decenio (2010).

En el contexto epidemiológico, la tendencia a la reducción de la TBC entre 1990-99 fué mayor (-7,8\% anual) que en el decenio 1981-90 $(-3,1 \%)$. En 2000 , la mortalidad alcanzó un nivel mínimo $(1,8 / 100.000 \mathrm{hbts})$ y se ha mantenido en esos bajos niveles. El último año informado (2003) se notificaron 2.908 casos de TBC en todas las formas, (tasa 18,4/100.000 hbts), de los cuales 1283 fueron bacilíferos (tasa 8,1/ $100.000 \mathrm{hbts}$ ). La enfermedad afecta más a hombres a partir de los 24 años y esta diferencia con respecto a las mujeres se amplia en los adultos mayores.

Respecto a los resultados terapéuticos, en cohortes de casos nuevos, pulmonares, bacilíferos adultos vírgenes a terapias previas (VT), la curación alcanzó al 86\%. Sin embargo, persiste aun un nivel alto de fallecidos $(7,6 \%)$, aunque una menor proporción de abandonos $(4,3 \%)$. Los fracasos son inusuales $(0,2 \%)$.

La estratificación muestra que los servicios con tasas < 20/100.000 corresponden a 11.181.934 hbts y representan 1.701 casos, con una tasa promedio de 15,2/100.000. Los más alejados del umbral de eliminación, con tasas sobre 25/100.000, corresponde a 7 servicios que comprenden 2.492 .606 hbts y representan 2.908 casos.

La eliminación de la tuberculosis como problema de Salud Pública es sin duda un objetivo deseable, atractivo y teóricamente factible, pero se hace cada vez más laboriosa a medida que sigue descendiendo la incidencia de la TBC.

La Organización Mundial de la Salud ha señalado los principales obstáculos en el camino hacia la eliminación de la tuberculosis en el mundo. Entre estos destacan:

- Falta de voluntad política para respaldar y mantener en funcionamiento los programas de control: "se eliminan los programas antes de eliminar la enfermedad".

- Creciente MultiDrogoResistencia, originada por programas deficientes.

- Mantención de altos niveles de pobreza y de extrema pobreza.

- No facilitar tratamiento gratuito a los enfermos tuberculosos. 
- Las poblaciones migratorias.

- La coinfección TBC/VIH-SIDA.

- Las reformas de los sistemas de salud.

- Fallas estructurales y de gestión de los programas de control.

El PCT de Chile ha sido afectado en grado variable por algunos de los factores numerados. Algunos de los que han tenido mayor relevancia en otros países, no han constituido hasta ahora, obstáculos mayores para el normal progreso de las actividades, ni han influído en su impacto epidemiológico. Nos referiremos a los siguientes:

a) Multirresistencia a las drogas antituberculosas $(M D R)$. El porcentaje de multirresistencia primaria en Chile es uno de los más bajos de América Latina $0,4 \%$. La vigilancia de la MDR es sin duda indispensable, aunque por ahora no constituye un problema mayor en Chile.

b) Coinfección $T B C / V I H$. Se estima que en la actualidad el Riesgo Anual de Infección tuberculosa en Chile sería menor de $0,19 \%$, con tasas de positividad del PPD de 3\% a los 15 años de edad y $6 \%$ a los 30 . Este desplazamiento de la edad de infección, debido a la declinación de la endemia y el incremento moderado de la infección VIH registrado en Chile en los últimos años, condicionarían una tasa de coinfección relativamente baja. De todos modos, se requiere actualizar los estudios de prevalencia de infección VIH en casos de tuberculosis.

c) Poblaciones migratorias. Se ha insistido mucho en el eventual impacto de la tuberculosis de ciudadanos extranjeros provenientes de países con situación epidemiológica más severa, sobre la endemia en Chile. En los últimos 5 años se han presentado alrededor de 220 casos acumulados en extranjeros, dentro de un total de 15.563 casos registrados en el país $(1,4 \%)$. Esta baja proporción indica que, por ahora, éste es un factor marginal.

En cambio, son relevantes tres factores en los resultados y perspectivas del PCT: la persistencia de la pobreza y la extrema pobreza, que afectan al $20 \%$ de la población chilena; las eventuales fallas de estructura y gestión del PCT, y la reciente Reforma de la Salud.

Respecto al impacto sobre el futuro del PCT derivado de las medidas que corresponden a la etapa actual del proceso de reforma y sobre sus repercusiones en la factibilidad de alcanzar las metas de eliminación de la tuberculosis, esto dependerá directamente de la forma en que sean puestas en práctica dichas medidas. Diversas interrogantes surgen, particularmente en relación con los Servicios de Atención Primaria, sobre la autonomía de los establecimientos hospitalarios y sobre la organización y funcionamiento de las autoridades sanitarias regionales.

Hasta ahora, durante todas las etapas cumplidas por el proceso de reforma en Chile, se ha tratado de mantener las actividades de control de la tuberculosis en todos sus aspectos: concepto de Programa; estructura, funciones, gratuidad, estrategias, definiéndolo en la terminología de la Reforma como un "bien público".

Otro de los mensajes importantes que se desea comunicar son los objetivos y metas del programa:

El PCT es un Programa de Salud Pública de alcance nacional, descentralizado, cuyas normas y operaciones técnicas se cumplen en todos los niveles de la estructura del Sistema Nacional de Servicios de Salud del país y en las Secretarias Regionales Ministeriales de Salud.

\section{Objetivo general}

Reducir significativamente el riesgo de infección de la tuberculosis como problema de Salud Pública.

\section{Meta sanitaria}

El Ministerio de Salud se ha propuesto alcanzar la meta de "Eliminación avanzada" de la tuberculosis, definida como morbilidad de 10/ 100.000 en el año 2008-2010 y la de "Eliminación como problema Salud Pública", con tasas de 5/100.000, en el año 2020.

\section{Objetivos específicos}

a) Lograr coberturas de vacunación BCG del $100 \%$ de los recién nacidos.

b) Localizar y diagnosticar sobre el $70 \%$ de la incidencia estimada de los casos pulmonares bacilíferos al examen directo de expectoración o al cultivo.

c) Lograr el $90 \%$ de curación de los casos pulmonares confirmados bacteriológicamente que ingresan al tratamiento con el esquema primario.

d) Efectuar un estudio completo en más de $90 \%$ de los contactos de casos de tuberculosis en cualquiera de sus formas, en menores de 14 años.

e) Mantener la tendencia decreciente de la incidencia de casos de tuberculosis en todas las formas, con una reducción anual del 7\% o más, con el fin de alcanzar la meta sanitaria en el plazo propuesto. 


\section{Población objetivo}

Toda la población es beneficiaria de las actividades del PCT, independientemente de su condición previsional. Por esto, sus acciones están integradas en el Sistema Nacional de Servicios de Salud y son gratuitas.

La población afiliada al sector privado de salud o beneficiaria de servicios e instituciones de salud dependientes de otros Ministerios (Gendarmería, Carabineros, Fuerzas Armadas), deberán ser atendidos en sus propias instituciones, con estricto cumplimiento de las Normas Técnicas del PCT, bajo el control y vigilancia de la Autoridad Sanitaria del PCT. Pero, si lo prefieren, podrán ser atendidos en los establecimientos que componen la red asistencial de los servicios de salud.

\section{Estructura}

La nueva legalidad establecida en Chile en el campo de la salud, tiene repercusiones importantes en la estructura y funciones del PCT. Las actividades de control de la tuberculosis tienen una estructura de tres niveles: central o ministerial; intermedia, integrada por las Secretarias Regionales Ministeriales y de los Servicios de Salud, y locales o de los establecimientos asistenciales.

En estos niveles las funciones más relevantes son:

- Nivel ministerial, normativo, de regulación y conducción nacional

- Nivel Regional, a cargo de la vigilancia epidemiológica y evaluación operacional regional. A este nivel corresponde el ejercicio de la Autoridad Sanitaria

- Nivel de Direcciones de Servicios, encargados de la coordinación y gerencia técnica operacional, y

- Nivel Local, encargado de la ejecución de las actividades en postas, consultorios, SAPUS, hospitales y unidades de urgencia adosados.

\section{Casos de TBC en la Región Metropolitana}

Sra. Zulena Torres, enfermera encargada de $T B C$ de la SEREMI

En los casos de TBC de la Región Metropolitana ingresados hasta el 30/06/05, destaca la diferencia que se produce entre el número de casos notificados respecto a los ingresados en el registro nacional. En este último registro existen menos casos, lo que depende de problemas administrativos, ya que en los servicios sin enfermera encargada del programa la notificación de casos es menor (SSMNorte).

En resumen hay 23 casos sin notificar de 383 casos ingresados al registro nacional, muchos de los cuales procederían del Instituto Nacional del Tórax, donde al parecer los médicos no estarían notificando todos los casos. En tanto, los casos notificados (ENO) alcanzarían a 471 casos y de estos 142 (30\%) pertenecerían a algún grupo de riesgo (85 adultos mayores, $15 \mathrm{VIH}, 15$ extranjeros, 15 indigentes y 12 reclusos).

\section{Octubre}

\section{Contribución de los grupos de riesgo (factor pobreza) \\ Dr. Carlos Peña M.}

Se analiza la contribución de los grupos de riesgo, en especial el factor pobreza e indigencia, en la incidencia de la tuberculosis y como determinantes de un menor grado de curación. Se mencionan los estudios presentados en el Congreso Europeo de Enfermedades Respiratorias 2005 en Copenhague, Dinamarca, donde se demostró nuevamente que el factor pobreza produce mayor abandono y también mayor mortalidad (Eur Respir J, 2005, 26 Suppl 49: 141-3), lo que obliga a implementar diversas estrategias de incentivos (dinero, alimentos, etc), como se hace en Rusia y otros países.

Esto reafirma la importancia de la investigación cooperativa que efectuaremos con el Control Diseases Center (CDC) de Atlanta, Georgia, USA, para conocer el efecto del nivel socioeconómico en la curación de la TBC en la Región Metropolitana.

\section{Noviembre}

\section{Tuberculosis Meníngea Infantil}

Se presenta un caso de TBC Meníngea Infantil del hospital San Borja-Arriarán. Se trata de una niña peruana, contacto de familiar bacilífero, en la cual se confirmó el diagnóstico con cultivo positivo del LCR. Afortunadamente se hizo un diagnóstico precoz, lo que contribuyó a evitar secuelas neurológicas.

Este caso enfatiza la necesidad de efectuar oportunamente los estudios de contactos en los grupos de riesgo. 


\section{Diciembre}

Organización de las actividades del Seminario Nacional de TBC

Dr. Manuel Zúñiga G.

Se organizaron las actividades del Seminario Nacional de TBC a efectuarse entre el 15 y 16 de Diciembre. Se decidió adoptar una orientación dinámica de trabajo grupal con participación de los SEREMIS Regionales. Se buscarán estrategias locales diseñadas para enfrentar los problemas regionales.

Se explorará a fondo el componente de grupos de riesgo ya que se tiene evidencia de su importancia: En el SSMC alrededor del 50\% de los casos pertenecen a estos grupos vulnerables; es el servicio con mayores tasas en la región Metropolitana. El Servicio de Salud Aconcagua tiene baja incidencia de TBC, pero todos ellos pertenecen a grupos de riesgo. Los países europeos con bajas tasas, también presentan un alto número de casos en estos grupos. Francia, por ejemplo, con inmigrantes, enfermos $\mathrm{VIH}(+)$ y ancianos en abandono o indigencia.

Se analizarán las diferencias comunales y el efecto de la pesquisa en recintos carcelarios, con la idea de planificar un programa de control dirigido a grupos vulnerables.
Otro de los parámetros a evaluar será la letalidad de la TBC, que en Chile alcanzó al 9,8\% entre 1999 y 2002 según el Depto. de Estadística del MINSAL (DEIS).

\section{Comentario Final}

Dr. Victorino Farga $C$.

Podrá llamar la atención que gran parte de las reuniones del Comité de Tuberculosis del Área Metropolitana hayan estado dedicadas, durante 2005, a revisar los ajustes administrativos derivados de la nueva ley del AUGE.

Las Reformas de la Salud, recientemente aprobadas por el gobierno, representan un desafío para los programas de Salud en Chile. La separación entre la Autoridad Sanitaria y los Servicios de Salud, llamados ahora Redes Asistenciales, está determinando cambios administrativos que afectarán, entre otros, al Programa de Control de la Tuberculosis, que tan exitoso ha sido hasta ahora en nuestro país.

Esto plantea el desafío de diseñar nuevas estrategias de colaboración y nuevas definiciones de roles y actividades, dentro de las cuales el PCT pueda mantener su integridad e incluso fortalecerse. 